[Deutsche Entomologische Zeitschrift 1889. Heft I.]

\title{
Zwitter und Monstrositäten aus den Sammlungen
}

von

Dr. L. v. Heyden und Dr. G. Kraatz.

(Hierzu Taf. I, Fig. 9-23.)

Fig. 9. Kopf von Hygrocarabus variolosus F. aus Ungarn mit normalem rechten und 10 gliederigem linken Fühler, eingesendet von Hrn. Giebeler. (Mus. Heyden.)

Fig. 10. Vorderleib eines vollständigen $Z$ witters von Lucanus cervus L. (von Reitter an Kraatz mitgetheilt) mit männlicher linker und weiblicher rechter Seite. (Mus. Kraatz.)

Fig. 11. Gnorimus nobilis L. mit vollständig verkrüppelten Fld., unter denen ebenfalls verkrüppelte Flügelstummel vorragen, aus Schlesien von Hrn. Major Gabriel mitgetheilt. (Mus. Kraatz.)

Fig. 12. Vorderkörper eines Laemostenus aus dem Caucasus (von Hrn. Reitter erhalten) mit einer bucklichen Auftreibung auf der linken Seite des Thorax. (Mus. Kraatz.)

Fig. 13. Geotrypes Typhoeus L. (aus der Berliner Gegend von Hrn. H. Klaeger mitgetheilt), Fühler mit doppeltem Knopf. (Mus. Kraatz.)

Fig. 14. Zonabris quadripuncta L. aus dem Caucasus (Reitter!) mit 2 fünfgliederigen Fühlerenden. (Mus. Heyden.)

Fig. 15. Fühler von Aromia moschata L. aus Darmstadt (Klingelhöffer!), bei dem sich das sechste Glied in zwei Aeste, einen 6 gliederigen und einen 3 gliederigen spaltet. (Mus. Heyden.)

Fig. 16. Fühler von Calosoma investigator L. (aus der Berliner Gegend von Hrn. Klaeger mitgetheilt), bei dem vom verstärkten fünften Gliede zwei Aeste ausgehen, von denen der rechte 5 gliederig, der linke 6 gliederig ist, an der Spitze aber wiederum getheilt, und 2 zweigliederige Aeste zeigt. (Mus. Kraatz.)

Fig. 17. Bein eines Carabus auratus L. (aus Halle von Hrn. Dr. Knauth in Dresden mitgetheilt), bei welchem eine Dreitheilung des Schenkels anzunehmen ist, aber nur zwei verkrüppelte Schienen vorhanden sind. (Mus. Kraatz.)

Fig. 18. Rechte Mittelschiene einer Taurhina Nireus Schaum aus dem Aschanti Gebiete (Reitter), welche zweitheilig ist, jeder Theil mit einer ausgebildeten Tarse versehen. (Mus. Kraatz.) 
Fig. 19. Zweitheilige Afterspitze einer Melolontha vulgaris F., welche in Interlaken von Hrn. v. Heyden gesammelt ist.

Fig. 20. Chrysomela fimbrialis Küster (von Hrn. Schulrath Schreitter aus Görz mitgetheilt), mit 2 stark entwickelten Rippen auf jeder Fld. (Mus. Kraatz.)

Fig. 21 a. Thorax von Hydrobius fuscipes L., dessen Seiten flügelartig erweitert sind; b) zeigt die gewöhnliche Bildung des Vorderleibes. Nassau Dr. Buddeberg in Mus. Heyden.

Fig. 22. Fühler von Melolontha vulgaris Fabr., mit einer dreieckigen Erweiterung in der Mitte des letzten Gliedes an jedem Fühler (aus der Berliner Gegend von Hrn. Klaeger mitgetheilt). (Mus. Kraatz.)

Fig. 23. Kopf von Cerambyx cerdo L. (aus Illyrien von Hrn. Schulrath Schreiber eingesandt), mit vollständig entwickeltem ersten Wurzelgliede neben dem gewöhnlichen Fühlergliede (Mus. Kraatz). Es kommt nur äufserst selten vor, dafs ein zweites Fühlerglied sich neben einem anderen zeigt; Mocquérys (Col. anormaux 1880) zählt keinen ähnlichen Fall auf; noch seltener sind drei Glieder neben einander (vergl. diese Zeitschr. 1877, p. 57, Fig. 22, Taf. I).

Die Aufsätze zu Tafel II sind in Heft II enthalten.

\section{Ueber Käfer von Eisleben.}

Philonthus Scribae Fauvel (varipennis Scriba) ist von Hrn. Goldfufs in einem, von mir verglichenen Ex. am salzigen See bei Eisleben aufgefunden worden. Derselbe machte mir die nicht uninteressante Mittheilung, dafs

Hydroporus depressus Fabr. am salzigen See von ihm und Anderen nicht unmittelbar im Salzwasser, sondern in der Nähe des Ufers im losen, kiesigen Boden in Menge gesammelt wurde.

Pogonus luridipennis und iridipennis wurden früher nach Taschenberg und Bach (Käferfauna) häufig am salzigen See beobachtet, halophilus dagegen seltener. Hr. Goldfufs fing seit fünf Jahren kein en luridipennis, iridipennis ziemlich selten, halophilus dagegen sehr häufig. Es wäre also darauf zu achten, ob dies nur zufällig geschehen, oder ob die eine Art die andere verdrängt hat, was wohl anzunehmen wäre, wenn luridipennis auch fernerhin fehlt.

Dr. G. Kratz. 


\section{$2 \mathrm{BHL}$ Biodiversity Heritage Library}

Heyden, Lucas von and Kraatz, G. 1889. "Zwitter und Monstrositäten aus den Sammlungen." Deutsche entomologische Zeitschrift 1889(1), 221-222. https://doi.org/10.1002/mmnd.48018890138.

View This Item Online: https://www.biodiversitylibrary.org/item/103677

DOI: https://doi.org/10.1002/mmnd.48018890138

Permalink: https://www.biodiversitylibrary.org/partpdf/235439

\section{Holding Institution}

Harvard University, Museum of Comparative Zoology, Ernst Mayr Library

\section{Sponsored by}

Harvard University, Museum of Comparative Zoology, Ernst Mayr Library

\section{Copyright \& Reuse}

Copyright Status: Public domain. The BHL considers that this work is no longer under copyright protection.

This document was created from content at the Biodiversity Heritage Library, the world's largest open access digital library for biodiversity literature and archives. Visit BHL at https://www.biodiversitylibrary.org. 